
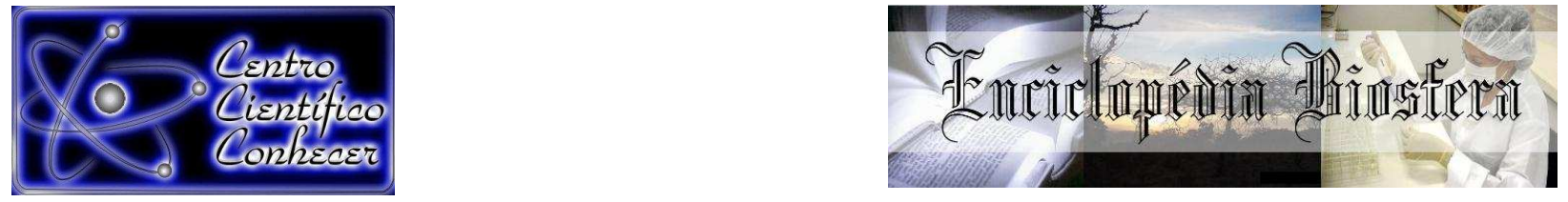

\title{
BIOMETRIA E EMERGÊNCIA DE PLÂNTULAS DE IPÊ DE JARDIM (TECOMA STANS) ORIUNDAS DE DIFERENTES PROCEDÊNCIAS DA REGIÃO METROPOLITANA DE BELÉM, PARÁ
}

Denise Nunes Pereira ${ }^{1 *}$, Ana Paula Ribeiro Medeiros ${ }^{1}$, Kelly da Conceição Pereira Santos ${ }^{1}$, Renata Sousa Tenório², Helaine Cristine Gonçalves Pires ${ }^{3}$.

1 Mestranda em Ciências Florestais da Universidade Federal da Amazônia, (dnizpereira@hotmail.com*) Belém, Pará, Brasil.2 Mestranda em Ciências Ambientais da Universidade Estadual do Pará, Belém, Pará, Brasil.

3 Doutoranda BIONORTE, Universidade Federal do Amazonas, Manaus, Amazonas, Brasil.

\section{Recebido em: 08/09/2015 - Aprovado em: 14/11/2015 - Publicado em: 01/12/2015} DOI: http://dx.doi.org/10.18677/Enciclopedia_Biosfera_2015_104

\begin{abstract}
RESUMO
A espécie Tecoma stans, vulgarmente conhecida como ipê de jardim, é arbustiva muito utilizada na ornamentação e arborização urbana por apresentar abundância de floração e fácil propagação. Este estudo teve como objetivo avaliar os parâmetros genéticos, para a posterior seleção dos indivíduos superiorizados de forma que sejam preditos parâmetros como ganho genético e genótipos melhorados da espécie. O experimento foi conduzido no viveiro do Instituto de Ciências Agrárias da Universidade Federal Rural da Amazônia (UFRA), em Belém-PA. Utilizou-se o delineamento experimental de blocos casualizados, com cinco tratamentos (procedência) e cinco repetições de 6 sementes por tratamento, totalizando 150 plantas no experimento. A germinação das mudas foi acompanhada diariamente através da contagem das plantas emergidas, ao longo de 15 dias, avaliando-se a primeira contagem de germinação no sétimo dia e a última contagem no $21^{\circ}$ dia. Os parâmetros de avaliação da germinação foram a porcentagem de germinação (\%G), o índice de velocidade de germinação (IVG), e o tempo médio de germinação (TMG). Foram medidas também a altura e o diâmetro do coleto (DC) e número de folhas. Não houve variação significativa entre as procedências quanto aos parâmetros de altura, diâmetro altura do colo (DC) e número de folhas, TMG e espessura da semente. O crescimento das plântulas depende do tamanho e da procedência das sementes.
\end{abstract}

PALAVRAS-CHAVE: arborização urbana; bignoniaceae; germinação.

\section{BIOMETRICS AND EMERGENCE OF SEEDLINGS OF GARDEN OF IPÊ (TECOMA STANS) SOURCE OF THE DIFFERENT PROVENANCES FROM REGION METROPOLITANA OF BELÉM, PARÁ}

\footnotetext{
ABSTRACT

The specie Tecoma stans, usually know as garden ipê, is shrubby very used urban in ornamentation and afforestation by presenting plenty of florewing and propagation easy. This study aimed to assess the genetic parameters for the subsequent selection of the higher individuals so that they are predicted parameters such as
} 
genetic gain and improved genotypes of species. The experiment was led in the Institute nursery of Agricultural Sciences of Universidade Federal Rural da Amazônia (Ufra), in Belém-PA. The experiment that was used experimental design of randomized blocks, with five treatments and five repeats of six seeds for treatment, totaling 150 plants in experiment. The germination of seedlings was accompanied daily through count emerged plants, over 15 days, evaluating yourself first count of germination in seventh day and last count in twenty-first day. The evaluation parameters of germination were percentage of germination (\%G), germination speed index (IVG) and mean germination time (TMG). Also measures were height and diameter of the coleto and the number of leaves. Hasn't signficant variation between provenances as the height parameters, diameter of the coleto and number of leaves, TMG and thickness of seed. The growth of seedlings it depends height and provenances of seeds.

KEYWORDS: Germination; urban afforestation; Bignoniaceae.

\section{INTRODUÇÃO}

Pertencente à família Bignoniaceae e vulgarmente conhecida como ipê de jardim, Tecoma stans (L.) Kunth, popularmente conhecida como amarelinho, falso ipê-amarelo, carobinha e ipê mirim, é uma espécie vegetal originária da região compreendida entre o México e o sul dos Estados Unidos (KRANZ \& PASSINI, 1997). O Ipê de jardim é uma espécie muito utilizada para a ornamentação de centros urbanos, arborização de ruas, praças e jardins, isto devido a beleza da sua floração, que além de tudo é abundante (BREDOW, 2007).

A capacidade de dispersão a curtas distâncias é comprovada por sua facilidade de propagação vegetativa, sendo que pesquisas mostraram $100 \%$ de enraizamento de estacas caulinares (BIONDI et al., 2008). A rapidez na dispersão da espécie tem sido associada com a produção de um grande número de sementes de tamanho pequeno e com prolongada viabilidade (BREDOW, 2007). No paisagismo é apresentada isolada ou em grupos, formando renques. No entanto sua utilização é controversa, pois apesar de ser muito ornamental é considerada uma perigosa planta invasora, capaz de inutilizar pastagens e prejudicar a regeneração de áreas degradadas. Isto se deve à sua grande capacidade de produzir sementes viáveis e ao seu rápido crescimento (PRATO, 2015).A respeito da influência da procedência das sementes sobre a germinação e emergência de plântulas pouco se sabe. Contudo, CALAI (2011) afirma que a procedência das sementes utilizadas é fator fundamental no planejamento da Arborização Urbana, pois define a variabilidade genética das mudas a serem implantadas. A germinação das sementes é regulada pela interação da sua qualidade fisiológica e das condições ambientais, sendo que cada espécie vegetal exige um conjunto de requisitos específicos quanto à disponibilidade de água, temperatura, luz e profundidade de semeadura para a ocorrência do processo de germinação de suas sementes (CARVALHO \& NAKAGAWA, 2012). O maior desempenho inicial de uma plântula é favorecida, conforme SCHUCH et al. (1999) e MUNIZZI et al. (2010) quando as sementes de alto vigor apresentam maior velocidade nos processos metabólicos, propiciando emissão mais rápida e uniforme da raiz primária no processo de germinação e maior taxa de crescimento.Diante do exposto, este trabalho objetivou estimar e quantificar indivíduos de ipê amarelo analisando-se os parâmetros genéticos, para a posterior seleção dos indivíduos superiorizados de forma que sejam preditos parâmetros como ganho genético e genótipos melhorados da espécie. 


\section{MATERIAL E MÉTODOS}

O experimento foi conduzido no viveiro do Instituto de Ciências Agrárias da Universidade Federal Rural da Amazônia (UFRA), em Belém-PA, com coordenadas geográficas de $01^{\circ} 27^{\prime} 25^{\prime \prime}$ e $01^{\circ} 27^{\prime} 39^{\prime \prime}$ latitude $S$ e $48^{\circ} 26^{\prime} 36^{\prime \prime}$ e 48 $26^{\prime} 48^{\prime \prime}$ de longitude WGr. O clima, segundo a classificação de Köppen é do tipo Af, ou seja, clima tropical chuvoso de monção, com a média anual da temperatura do ar de 26,0 $\pm 0,4 \stackrel{\circ}{\circ} \mathrm{C}$, com máximas e mínimas variando de $31,5 \pm 0,7 \stackrel{\circ}{\circ} \mathrm{C}$ a $22,0 \pm 0,3{ }^{\circ} \mathrm{C}$, respectivamente, durante o ano. A pluviosidade média anual é de $2.858,7 \pm 76,6$ $\mathrm{mm} /$ ano com maior volume no período chuvoso (dezembro a maio), correspondendo a $71,2 \%$ do total anual, enquanto que os $28,8 \%$ restantes são distribuídos nos meses de junho a novembro (SILVA JUNIOR et al., 2012).

As sementes de Ipê de jardim (Tecoma stans) foram coletadas de cinco procedências da região metropolitana de Belém, nos seguintes bairros: Pedreira, Cidade Nova, Guanabara, Montese e Marco (Quadro 1).

QUADRO 1. Localização e coordenadas geográficas de coleta dos frutos das árvores matrizes de Tecoma stans. Belém- PA.

\begin{tabular}{|c|c|c|}
\hline \multirow{2}{*}{$\begin{array}{c}\text { LOCAL } \\
\text { (Matrizes) }\end{array}$} & \multicolumn{2}{|c|}{ COORDENADAS GEOGRÁFICAS } \\
\hline & LATITUDE & LONGITUDE \\
\hline PEDREIRA & 125’09.6" & $4827 ’ 58.4 "$ \\
\hline CIDADE NOVA & 121'21.3" & 4824 '05.9" \\
\hline GUANABARA & $1233^{\prime} 38$. & 4825'13.3" \\
\hline MONTESE & 126'02" & 4827'10.6" \\
\hline MARCO & $126 \prime 11.9 "$ & 4828'40.9" \\
\hline
\end{tabular}

Após a coleta, os frutos do ipê de jardim, separados por local, foram triados e as sementes, separadas em um lote homogêneo, de acordo com o tamanho, sendo eliminadas aquelas atacadas por patógenos e com má formação. Após o beneficiamento, as dimensões das sementes (comprimento, largura e espessura) foram determinadas com o auxílio de paquímetro digital.

A semeadura foi realizada em sacos plásticos de dimensões de $10 \mathrm{~cm} \times 20$ $\mathrm{cm}$, nos quais foi colocada uma mistura de substrato de terra preta com casca de castanha, de forma individualizada, colocando-se uma semente por embalagem.

Utilizou-se o delineamento experimental de blocos casualizados, com cinco tratamentos (procedência) e cinco repetições de 6 sementes por tratamento, totalizando 150 plantas no experimento. As mudas foram conduzidas em ambiente parcialmente sombreado, sendo realizada diariamente irrigação das mudas no inicio da manhã e fim da tarde. Como forma de diminuir a competição entre as plantas foi retirada manualmente as plantas invasoras que germinaram na embalagem.

A germinação das mudas foi acompanhada diariamente através da contagem das plantas emergidas, ao longo de 15 dias, avaliando-se a primeira contagem de germinação no sétimo dia e a última contagem no $21^{\circ}$ dia. Os parâmetros de avaliação da germinação foram à porcentagem de germinação $(\% \mathrm{G})$, o índice de velocidade de germinação (IVG), e o tempo médio de germinação (TMG), conforme MAGUIRE (1962).

Foi determinada também, a altura $(\mathrm{H})$, número de folhas $(\mathrm{NF})$ e diâmetro do coleto da planta (DC), sendo a altura, medida com uma régua, considerado do solo 
até a parte mais alta da planta e o diâmetro do coleto determinado por um paquímetro digital com precisão de 0,01 mm (JASPER et al., 2006). Todos os resultados foram submetidos à ANOVA e comparados pelo teste de Tukey ao nível de $5 \%$ de probabilidade com o uso do Programa SISVAR ${ }^{\circledR}$ (FERREIRA,2007).

\section{RESULTADOS E DISCUSSÃO}

Observou-se que os frutos coletados em forma de vagem no bairro da Pedreira eram maiores e mais homogêneos em comparação aos encontrados no bairro da Montese que apresentavam menor comprimento e grande variação na espessura (Figura 1).

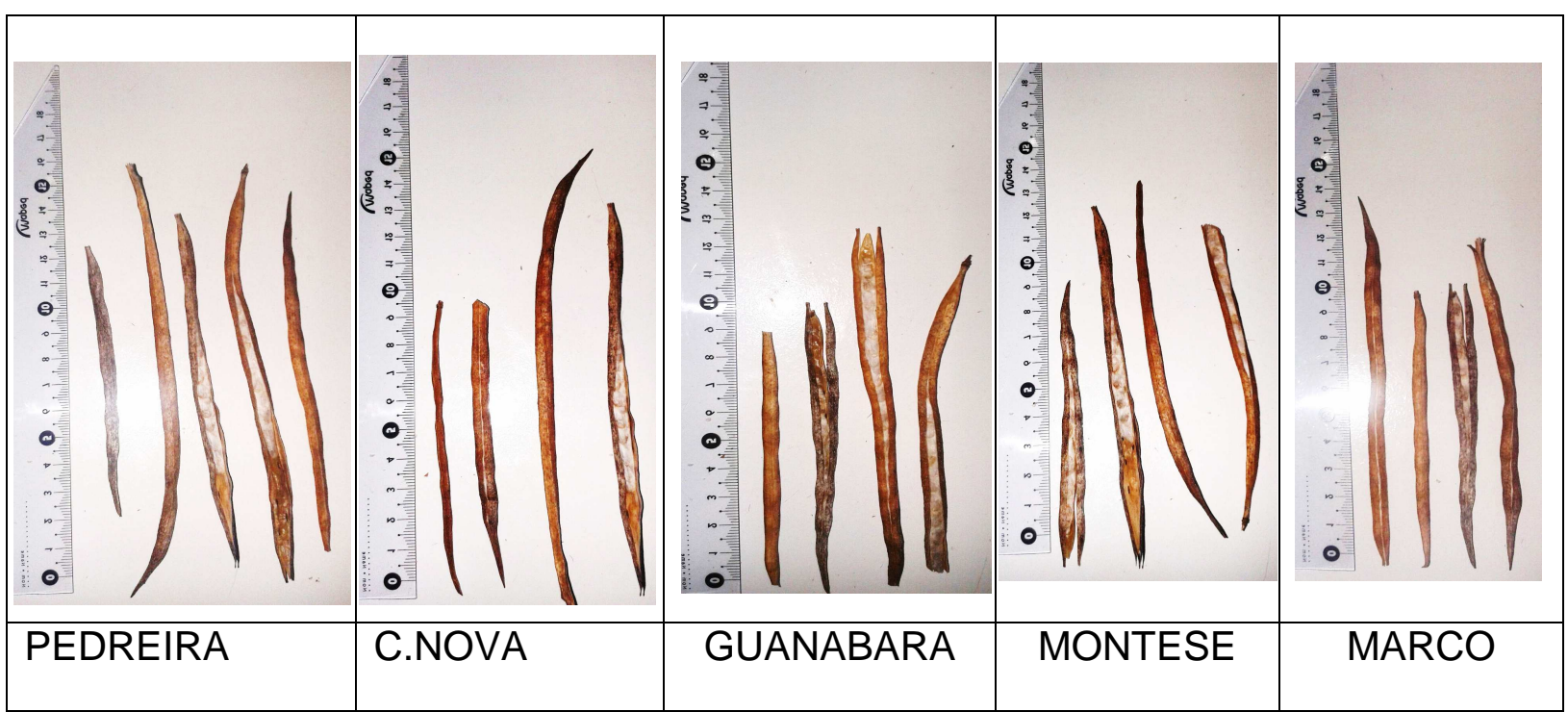

FIGURA 1. Frutos de ipê de jardim coletados em diferentes procedências da regiãometropolitana de Belém, $\mathrm{Pa}$.

Em relação às sementes, o comprimento e a largura das coletadas na Pedreira e Cidade Nova foram maiores que as das demais procedências, no entanto para a variável largura $(\mathrm{mm})$ os valores não apresentaram diferenças significativas para as sementes coletadas no bairro da Guanabara, sendo o menor valor encontrado no bairro do Marco. As espessuras de sementes de ipê de jardim apresentaram não significância pelo teste de Tukey a $5 \%$ de probabilidade. De maneira geral as sementes de maior tamanho médio foram encontradas na Cidade Nova e as menores no bairro Montese (Tabela 1). Isto deve-se ao cuidado exercido pelos próprios moradores ao redor da matriz da Cidade Nova em executar alguns tratos culturais além da adubação. Por isso, observa-se a importância da condução dos arbustos para que de forma eficaz a arborização urbana com essa espécie atinja níveis satisfatórios de aceitação pela capacidade de ofertar sombra, beleza e sementes para propagação. 
TABELA 1 Características físicas de sementes de Ipê de Jardim (Tecoma stans) de diferentes procedências da região metropolitana de Belém, Pa.

\begin{tabular}{ccccc}
\hline Procedência/Características & $\begin{array}{c}\text { Comprimento } \\
(\mathbf{m m})\end{array}$ & $\begin{array}{c}\text { Largura } \\
(\mathbf{m m})\end{array}$ & $\begin{array}{c}\text { Espessura } \\
(\mathbf{m m})\end{array}$ & $\begin{array}{c}\text { Tamanho } \\
\text { médio } \\
(\mathbf{m m})\end{array}$ \\
\hline Pedreira & $23,57 \mathrm{a}$ & $7,99 \mathrm{a}$ & $4,29 \mathrm{a}$ & 11,95 \\
Cidade Nova & $25,48 \mathrm{a}$ & $7,52 \mathrm{ab}$ & $4,85 \mathrm{a}$ & 12,62 \\
Guanabara & $19,48 \mathrm{~b}$ & $7,51 \mathrm{ab}$ & $4,54 \mathrm{a}$ & 10,51 \\
Montese & $16,64 \mathrm{~b}$ & $6,81 \mathrm{cb}$ & $4,93 \mathrm{a}$ & 9,45 \\
Marco & $18,016 \mathrm{~b}$ & $6,148 \mathrm{c}$ & $4,69 \mathrm{a}$ & 9,62 \\
\hline
\end{tabular}

Médias seguidas de mesma letra, na coluna não diferem estatisticamente pelo teste Tukey a $5 \%$.

Os parâmetros genéticos são apresentados na Tabela 2, onde as sementes das diferentes procedências variaram em média de $44 \%$ a $88,8 \%$ para a taxa de germinação, com tempo médio de germinação de 11 dias para todas as procedências e IVG entre 0,374 e 0,602. Houve diferença significativa a $5 \%$ de probabilidade, entre as procedências, para as variáveis \%G e IVG. Para um programa de melhoramento genético, essa variabilidade observada torna-se condição essencial para o estabelecimento do programa além de seu maior desenvolvimento em estudos futuros (COSTA et al., 2007).

A procedência com maior taxa de germinação foi a encontrada no bairro do Marco, que também apresentou alto índice de velocidade de germinação. Para o bairro Pedreira ocorreu a menor valor de \%G e IVG com média de 11 dias para germinação das sementes. A explicação dos dados obtidos deve-se às características biométricas das sementes serem variáveis em função das condições ambientais durante sua formação, além das características genéticas das matrizes (DUARTE et al., 2012).

TABELA 2 Taxa de Germinação (G), Tempo médio de germinação (TMG), Índice de Velocidade de Germinação (IVG) de sementes de Ipê de Jardim (Tecoma stans) de diferentes procedências da região metropolitana de Belém, Pa.

\begin{tabular}{cccc}
\hline Procedência de Ipê de jardim & G (\%) & TMG (dias) & IVG \\
\hline Pedreira & $44 \mathrm{~b}$ & $11,4 \mathrm{a}$ & $0,37 \mathrm{~b}$ \\
Cidade nova & $84,8 \mathrm{a}$ & $11,0 \mathrm{a}$ & $0,57 \mathrm{a}$ \\
Guanabara & $81,6 \mathrm{a}$ & $11,0 \mathrm{a}$ & $0,57 \mathrm{a}$ \\
Montese & $55,6 \mathrm{a}$ & $11,4 \mathrm{a}$ & $0,428 \mathrm{~b}$ \\
Marco & $88,8 \mathrm{a}$ & $11,0 \mathrm{a}$ & $0,602 \mathrm{a}$ \\
\hline
\end{tabular}

Médias seguidas de mesma letra, na coluna não diferem estatisticamente pelo teste Tukey a $5 \%$.

ROSA et al. (2011) ao testarem o potencial de germinação de 200 sementes da espécie Attalea maripa, levando em consideração o critério de separação das sementes que apresentaram melhor estado e qualidade fisiológica, concluíram que o 
peso, comprimento e diâmetro médio obtidos foram: $26,91 \mathrm{~g}, 56,04 \mathrm{~cm}$ e $30,20 \mathrm{~cm}$, respectivamente.

O início do processo germinativo ocorreu no $13^{\circ}$ dia após instalação do experimento e, estabilizou-se a partir do $210^{\circ}$ dia. As maiores porcentagens de germinação foram observadas nas sementes coletadas no bairro do Marco $(88,8 \%)$ e Cidade Nova (84,8\%), respectivamente. As menores porcentagens foram as coletadas no bairro da Pedreira (44\%) e Montese (55,6\%). Dessa forma, os tratamentos que apresentaram sementes de baixo potencial germinativo corroboram com a importância dos parâmetros genéticos à seleção de sementes a fim de obterse elevada produção de mudas e, posteriormente, indivíduos sadios e livre de doenças.

Os dados de altura, diâmetro do coleto (DC) e número de folhas não apresentaram diferença significativa, pelo teste de Tukey a $5 \%$ de probabilidade, para nenhuma procedência estudada, indicando que não há diferença no desempenho das progênies para estas características. As mudas que alcançaram maiores alturas foram do bairro da Cidade Nova e Pedreira com 8,06 cm e 6,28 cm, respectivamente. Para o DC a procedência da Pedreira e Guanabara apresentaram os maiores valores, de $1,4 \mathrm{~mm}$ e $1,25 \mathrm{~mm}$, respectivamente (Tabela 3 ). Esses valores podem ser explicados pelo maior número de folhas encontrado nas mudas dessas procedências, uma vez que o espaçamento estabelecido entre as mudas favoreceu o crescimento em diâmetro pela redução da competição intraespecífica (FERREIRA, et al., 2012).

TABELA 3 Altura da planta $(\mathrm{H})$, diâmetro do coleto (DC) e número de folhas (NF) de Ipê de Jardim (Tecoma stans) de diferentes procedências da região metropolitana de Belém, Pa.

\begin{tabular}{cccc}
\hline Procedência de Ipê de jardim & H $(\mathbf{c m})$ & DC $(\mathbf{m m})$ & NF \\
\hline Pedreira & $6,28 \mathrm{a}$ & $1,402 \mathrm{a}$ & $8,2 \mathrm{a}$ \\
Cidade nova & $8,06 \mathrm{a}$ & $1,226 \mathrm{a}$ & $7,6 \mathrm{a}$ \\
Guanabara & $5,08 \mathrm{a}$ & $1,256 \mathrm{a}$ & $6,4 \mathrm{a}$ \\
Montese & $5,7 \mathrm{a}$ & $1,178 \mathrm{a}$ & $6,2 \mathrm{a}$ \\
Marco & $4,44 \mathrm{a}$ & $0,976 \mathrm{a}$ & $5,8 \mathrm{a}$ \\
\hline
\end{tabular}

Médias seguidas de mesma letra, na coluna não diferem estatisticamente pelo teste Tukey a $5 \%$.

\section{CONCLUSÃO}

A procedência das sementes influencia nas características biométricas e germinação. As procedentes do bairro da Cidade Nova apresentam o maior vigor e desempenho germinativo.

\section{REFERÊNCIAS}

BIONDI, D.; BREDOW, E.A.; LEAL, L. Influencia do diâmetro de estacas no enraizamento de Tecoma stans (L.) Juss. ex Kunt. Semina: Ciências Agrárias, Londrina, v. 29, n.2, p. 277-282,2008. 
CALAI, F. A. ESTÁGIO CURRICULAR OBRIGATÓRIO SUPERVISIONADOSEÇÃO VIVEIRO. UNIVERSIDADE FEDERAL DO RIO GRANDE DO SUL, 2011.CARVALHO, N.M.; NAKAGAWA, J. Sementes: ciência, tecnologia e produção. 5.ed. Jaboticabal: FUNEP, 2012. 590p.

COSTA, B.R.; ROA, R.A.R.; XAVIER, T.M.; FLORES, H.C.G. Desenvolvimento inicial de progênies de Ipê Amarelo (Tabebuia Chrysotricha (Mart. ex A. Dc.) Standl.) no Município de Campo Grande, MS, Brasil. Ensaios E Ci., Campo Grande, V. 11, N. 2, P.39-45, Ago. 2007.

DUARTE, R. I; SILV, F. A.L.S.;SCHULTZ, J. SILVA, J.Z.; REIS, M. S. Característica de desenvolvimento inicial em teste de progênie de uma população de Araucária na Flona de Três Barras- SC. Instituto Chico Mendes Biodiversidade Brasileira 2(2), 114-123, 2012.

FERREIRA, D.F. Programa Sisvar versão 5.1. Lavras: Departamento de Ciências Exatas da Universidade Federal de Lavras, 2007.

FERREIRA, R.A.;WINCKLER, D.C.F.;SILVA, J.M.S. Efeito de diferentes espaçamentos no desenvolvimento de mudas do triclos Eucalyptus urophylla vs. Eucalyptus grandis vs. Eucalyptus globulus em viveiro. Re.C.E.F., v.19, n.1, fev, 2012.

JASPER,R.;JASPER,M.;GARCIA, L.C. Seleção de sementes de milho durante a simulação da semeadura com disco perfurado horizontal. Eng. Agríc. vol.26 no.2 Jaboticabal May/Aug. 2006.

KRANZ, W.M. \& T. PASSINI. 1997. Amarelinho - Biologia e controle. Informe da Pesquisa, Londrina, IAPAR 17, 19p.

MAGUIRE, J.D. Speed of germination-aid in selection and evaluation for seedlig emergence and vigor. Crop Science, Madison, v.2, n.1, p.176-177,1962.

MUNIZZI, A; BRACCINI.; A.L.; RANGEL, MA. S; SCAPIM; CA; ALBRECHT, L.P. Qualidade de sementes de quatro cultivares de soja, colhidas em dois locais no estado de Mato Grosso do Sul. Revista Brasileira de Sementes: v.32, n.1, p.176$185,2010$.

PATRO, R. Ipê-de-jardim - Tecoma stans. Jardineiro. net. Disponivel em:< http://www.jardineiro.net/plantas/ipe-de-jardim-tecoma-stans.html>. Acesso em: 06 de setembro de 2015.

ROSA, L dos S; FERREIRA, P. R. N; SILVA, V. M; NOGUEIRA, G. A. dos S; CARMO, W. M. Biometria e germinação de Attalea maripa (Aubl.) mart. (inajá) em diferentes substratos. In. Congresso Brasileiro de Sistemas Agroflorestais, Belém- PA, 2011. 
SCHUCH, L.O.B.; NEDEL, J.L.; ASSIS, F.N. Crescimento em laboratório de plântulas de aveia-preta (Avena strigosa Schreb.) em função do vigor das sementes. Revista Brasileira de Sementes, v.21, n.1, p.229-234, 1999.

SILVA JÚNIOR, J. A.; COSTA, A. C. L.; PEZZUTI, J. C. B.; COSTA, R. F.; GALBRAITH, D. Análise da distribuição espacial do conforto térmico na cidade de Belém, PA no período menos chuvoso. Revista Brasileira de Geografia Física, v.2, p.218-232, 2012. 